\title{
FORMAL INVERSION OF THE SELF-CONSISTENT PROBLEM FOR TRIAXIAL GALAXIES
}

\author{
Herwig Dejonghe \\ The Institute for Advanced Study \\ Princeton, NJ 08540
}

Triaxial separable potentials $V(\lambda, \mu, \nu)=f_{\lambda}+f_{\mu}+f_{\nu} \geq 0$, with

$$
f_{\lambda}=\frac{f(\lambda)}{(\lambda-\mu)(\lambda-\nu)}, \quad f_{\mu}=\frac{f(\mu)}{(\mu-\nu)(\mu-\lambda)}, \quad f_{\nu}=\frac{f(\nu)}{(\nu-\lambda)(\nu-\mu)},
$$

admit three constants of the motion (see de Zeeuw 1985 for more details), for which we can take the following set:

$$
E=\sum_{c y c}\left(f_{\lambda}-\frac{1}{2} v_{\lambda}^{2}\right), \quad J=\sum_{c y c}(\mu+\nu)\left(f_{\lambda}-\frac{1}{2} v_{\lambda}^{2}\right), \quad K=\sum_{c y c} \mu \nu\left(f_{\lambda}-\frac{1}{2} v_{\lambda}^{2}\right) .
$$

Here $v_{\lambda}, v_{\mu}$ and $v_{\nu}$ are the velocity components in the $(\lambda, \mu, \nu)$ coordinate system, and we recognize in $E$ the binding energy per unit mass.

We define the mass density $\rho(\lambda, \mu, \nu)$ as follows

$$
\rho(\lambda, \mu, \nu)=\iiint F(E, J, K) d v_{\lambda} d v_{\mu} d v_{\nu}
$$

The inversion problem has as its goal to determine $F(E, J, K)$ out of $\rho(\lambda, \mu, \nu)$. We have developed a formalism that establishes explicit relations in both directions between $F(E, J, K)$ and a function $\tilde{\rho}$ of six variables. It is always possible to recover the physical $\rho(\lambda, \mu, \nu)$ out of this function, but many different $\tilde{\rho}$ 's give the same $\rho(\lambda, \mu, \nu)$.

Assume that the distribution function $F(E, J, K)$ can be written as a combined Laplace-Fourier transform

$$
F(E, J, K)=-\frac{1}{2 \pi i} \int_{a_{0}-i \infty}^{a_{0}+i \infty} e^{a E} d a \frac{1}{2 \pi} \int_{-i \infty}^{+i \infty} \int^{b J+c K} d b d c \mathcal{F}(a, b, c),
$$

with $\Re\left(a_{0}\right)>0$. Performing the integration (3), we find $\rho$ as an integration over $a$, $b$ and $c$ of $d \rho$, given by

$$
d \rho=(2 \pi)^{3 / 2} \mathcal{F}(a, b, c)\left(\prod_{c y c} \frac{1}{\sqrt{a+b(\mu+\nu)+c \mu \nu}}\right) e^{a V+b W+c U} d a d b d c,
$$


with

$$
V=\sum_{c y c} f_{\lambda}, \quad W=\sum_{c y c}(\mu+\nu) f_{\lambda}, \quad U=\sum_{c y c} \mu \nu f_{\lambda} .
$$

The kernel in equation (5a) can be seen to permit a simpler form of (5):

$$
2^{3 / 2}\left(\prod_{c y c} \int_{-\infty}^{f_{\lambda}} \frac{d f_{\lambda}^{\prime}}{\sqrt{f_{\lambda}-f_{\lambda}^{\prime}}}\right) F(V, W, U)=\tilde{\rho}(V, W, U, \lambda, \mu, \nu),
$$

which is a product of three separated Abel integral equations. Hence, we immediately get the inversion of (6):

$$
F(V, W, U)=\frac{1}{2^{3 / 2} \pi^{3}}\left(\prod_{c y c} \int_{-\infty}^{f_{\lambda}} \frac{d f_{\lambda}^{\prime}}{\sqrt{f_{\lambda}-f_{\lambda}^{\prime}}} \frac{\partial}{\partial f_{\lambda}^{\prime}}\right) \tilde{\rho}\left(f_{\lambda}^{\prime}, f_{\mu}^{\prime}, f_{\nu}^{\prime}, \lambda, \mu, \nu\right) .
$$

We remark that the mass density is written as an abstract function of 6 variables instead of only the three coordinates. We call it the augmented mass density $\tilde{\rho}$. Obviously, when we substitute the values of $f_{\lambda}, f_{\mu}$ and $f_{\nu}$, as given by (1), into $\tilde{\rho}$, we find the usual $\rho(\lambda, \mu, \nu)$. We have assumed that $\rho\left(f_{\lambda}, f_{\mu}, f_{\nu}, \lambda, \mu, \nu\right)$ is zero at the boundary to sufficient order. The lower bound of the integrals in (5) and (6) are here formally $-\infty$, but must be assumed to coincide with the boundary of the model in 6-dimensional space.

Not all forms $\rho\left(f_{\lambda}, f_{\mu}, f_{\nu}, \lambda, \mu, \nu\right)$ lead, after performing (7), to a distribution function that is a function of three variables $V, W$ and $U$ only, and not of the six $f_{\lambda}, f_{\mu}, f_{\nu}, \lambda, \mu, \nu$. It is found that $\rho\left(f_{\lambda}, f_{\mu}, f_{\nu}, \lambda, \mu, \nu\right)$ should obey three cyclic partial differential equations of the form

$$
\begin{aligned}
\frac{\partial^{3} \tilde{\rho}}{\partial \nu \partial f_{\lambda} \partial f_{\mu}} & +\frac{1}{\lambda-\nu} \sqrt{f_{\lambda}} \frac{\partial}{\partial f_{\lambda}}\left[\sqrt{f_{\lambda}} \frac{\partial}{\partial f_{\mu}}\left(\frac{\partial \tilde{\rho}}{\partial f_{\lambda}}-\frac{\partial \tilde{\rho}}{\partial f_{\nu}}\right)\right] \\
& +\frac{1}{\mu-\nu} \sqrt{f_{\mu}} \frac{\partial}{\partial f_{\mu}}\left[\sqrt{f_{\mu}} \frac{\partial}{\partial f_{\lambda}}\left(\frac{\partial \tilde{\rho}}{\partial f_{\mu}}-\frac{\partial \tilde{\rho}}{\partial f_{\nu}}\right)\right]=0 \quad \text { (cyc.). }
\end{aligned}
$$

The construction of explicit dynamical models for triaxial systems hinges critically on our ability to find a solution $\tilde{\rho}$ for (8) that reduces to a given $\rho$. However, special solutions of (8) can be found for which the inversion (7) takes a particularly simple Eddington form. They are generalizations of the Osipkov-Merritt models. Moreover, (8) is a natural starting point--within this analysis-for a study of the non-uniqueness of the inversion problem.

The augmented mass density carries all the dynamical information, since the distribution function can be calculated from it. Hence, expressions can be given for all the moments as functionals of the augmented mass density. E.g., for the velocity dispersions we find the cyclic relations

$$
\tilde{\rho} \tilde{\sigma}_{\lambda}^{2}=\int^{f_{\lambda}} \tilde{\rho} d f_{\lambda}^{\prime}, \quad \tilde{\rho}=\frac{\partial}{\partial f_{\lambda}}\left(\tilde{\rho} \tilde{\sigma}_{\lambda}^{2}\right)
$$

\section{REFERENCE}

de Zeeuw, P.T., 1985. Mon. Not. R. Astr. Soc., 216, 273. 